\title{
Please, Help Me! I Am Lost in Zoom
}

\author{
Guillaume Touya $^{\mathrm{a} *}$, María-Jesús Lobo ${ }^{a}$, William A. Mackaness ${ }^{\mathrm{b}}$, Ian Muehlenhaus ${ }^{\mathrm{c}}$ \\ ${ }^{a}$ LASTIG, Univ Gustave Eiffel, ENSG, IGN, F-94160 Saint-Mandé, France, firstname.lastname@ign.fr \\ ${ }^{b}$ Institute of Geography, School of Geosciences, University of Edinburgh, Edinburgh, UK, dr.w.mackaness@ed.ac.uk \\ ${ }^{c}$ Division of Continuing Studies, University of Wisconsin-Madison, United States, muehlenhaus@wisc.edu \\ * Corresponding Author
}

\begin{abstract}
LostInZoom is a new research project that will seek to design novel ways of zooming into multi-scale maps, to overcome the desert fog effect that occurs with current multi-scale cartography techniques. The desert fog effect makes you feel lost for a few seconds after a zoom in or out, because the map has changed. The idea developed in this project is to propose anchor-based zooming techniques, where salient landmarks salient at multiple scales serve as anchors between maps during the zoom. This paper discusses the main challenges that will be addressed in this project: (1) better understand and measure the desert fog effect with maps; (2) defining and modelling the best anchors for anchor-based zooming; (3) designing more progressive multi-scale maps to host these anchors; (4) designing staged animations based on anchors between maps at different scales.
\end{abstract}

Keywords: scale, zoom, landmarks, map generalisation, Gestalt

\section{Introduction}

LostInZoom is a starting research project about zooming interactions in multi-scale interactive (Roth, 2013) 2D maps. This short paper presents the project rationale and reviews the research challenges that the project seeks to address.

Being lost in a zoom is a common experience of all users of multi-scale maps, regardless of spatial acuity or sense of direction. When you zoom in or out a multi-scale map, the map changes with scale, and it takes a few seconds to know exactly where you are looking on a map and where the place you are looking for is in comparison. Often, you may need to zoom in and out and back and forth a few times to determine where you are in the current map scale. This phenomenon is not limited to multi-scale maps, but common to most of the multi-scale interactive visualisations. Researchers in human-computer interaction call this phenomenon the desert fog (Jul and Furnas, 1998, Touya, 2019), as the zoom removes all, or most of the visual cues of your past location, just like a real desert fog.

The goal of the LostInZoom project is to design, implement and test novel solutions to overcome or mitigate desert fog. This we call anchor-based zooming (Figure 1). Anchorbased zooming replaces the current zoom animation with an animation where salient landmarks - i.e., graphic anchors that are visible across multiple scales - are highlighted during the scale change. The potential efficacy of anchor-based zooming is inspired by Anchor Theory (Couclelis et al., 1987), which states that our mental representation of space is anchored by a hierarchy of landmarks.

Designing anchor-based zooming techniques raises many research challenges that are discussed in the remainder of the paper.

\section{The Desert Fog Effect in Multi-Scale Maps}

The LostInZoom research project's first challenge is to measure, qualify, and quantify this desert-fog effect on multi- scaled maps. Our research will explore:

- Does desert fog occur with any level of scale change?

- Is desert fog more prominent when the scale change is significant?

- Does desert fog severity correlate with map style or how the map is generalised? We would assume that a good generalisation and consistent styles over scales would mitigate the desert fog.

For this first challenge, and most of the following ones, we will adopt an experimental approach with controlled user surveys and controlled user studies (Roth et al., 2017).

\section{Multi-Scale Landmark as Anchors in the Zoom}

The first step when designing an anchor-based zoom capable map is to better understand what types of objects work as efficient anchors. We will combine psychology-based user surveys, with eye-tracking analyses and content analysis of the maps, to understand what features in the maps are used consciously and subconsciously by map readers to know where they are during and after a zoom. Our hypothesis is that some anchors are personal, related to the personal experiences of map readers with the places depicted in the map. Couclelis et al. (1987) demonstrated that anchors in mental maps are related to experiences in the real world (e.g., home and workplace). Other anchors, however, should be common referents for a vast majority of users due to their visual saliency or their importance in the map. For instance, the Seine river is prominent in the maps of Paris. This research is an opportunity to revisit previous research on landmarks (Sorrows and Hirtle, 1999), usually dedicated to landmarks in the real world, rather than on a map. Past research showed the usefulness of artificial anchors such as grids, to enhance spatial memory performance (Dickmann et al., 2017), and knowing where you are during and after a zoom does make use 

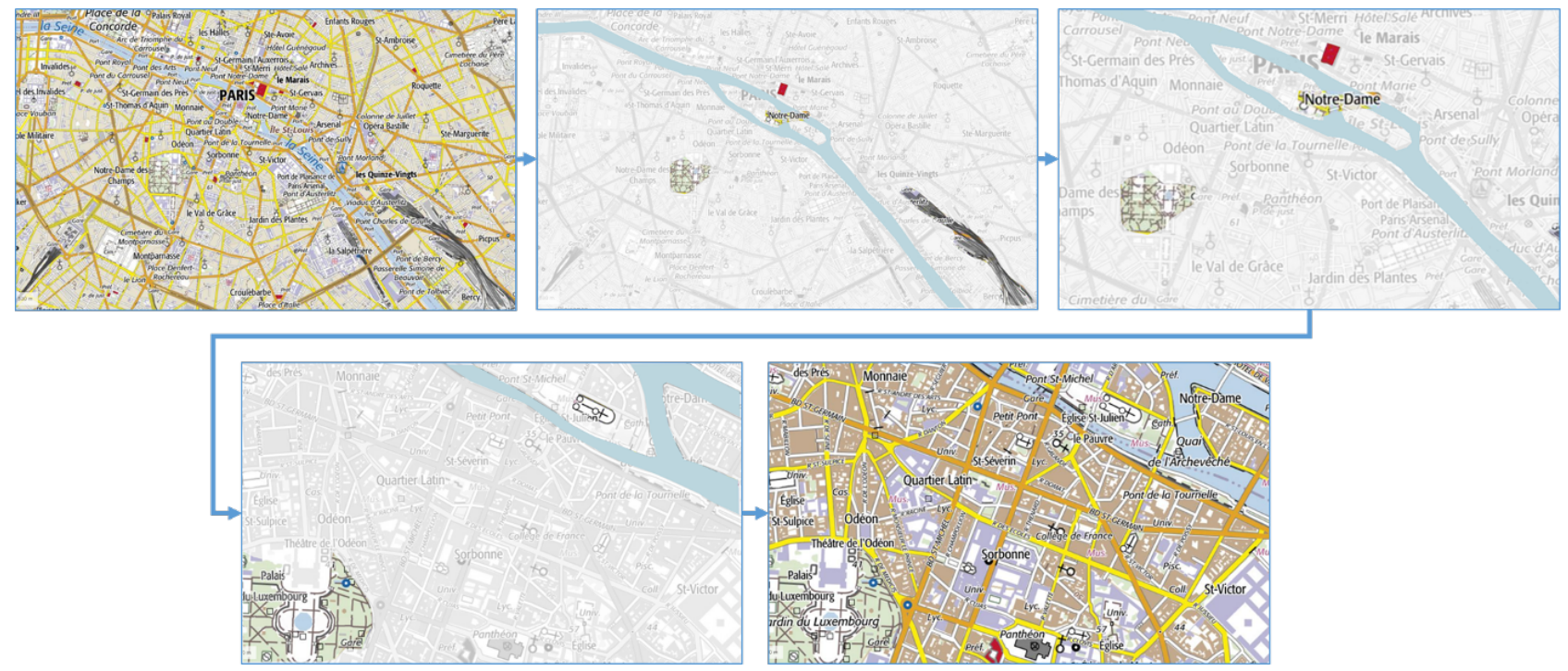

Figure 1. Montage that shows the different steps of an anchor-based zoom in the center of Paris: several landmarks in the map serve as anchors between the scales.

of spatial memory (Bestgen et al., 2017). We also plan to research how real map features (e.g., a bend in the river) perform as anchors versus different types of artificial anchors (e.g., a grid mark).

Multi-scale anchors based on landmarks must be salient at more than one scale; they need to be recognizable across many different scales, even if they are represented - or abstracted - differently. Our research will need to determine which characteristics of multi-scale anchors are most salient and important for clear and quick recognition. Knowing this will help cartographers better identify the anchors when making their own maps, and enhance anchor rendering across scales. Anchors can be a simple feature that are salient due to their importance, or due to the geographic context. For example, a summit in the middle of a flat landscape is salient because of context, while Mont Blanc is salient because it is the highest summit in Europe. But anchors can also be established via spatial relations between features (Touya et al., 2014), or among a group of features following a salient pattern (Mackaness and Edwards, 2002) such as the Paris ring road (Figure 2): these salient ring roads are groups of connected road sections surrounding a city, and connecting major roads to the city street network. We will analyze and scrutinize the characteristics of anchors identified by map users, in terms of Gestalt principles of perceptual grouping, to understand what makes them so recognisable. Clutter (Rosenholtz et al., 2007) is another interesting aspect: are anchors as effective in very cluttered maps?

The following challenge is to automatically detect anchors in maps. The recognition of specific patterns and structures in a map is not a new challenge, and spatial analysis (Heinzle and Anders, 2007), or even ontologies (Lüscher et al., 2008) can be used in the recognition process. In this latter case, a multi-scale ontology (Carral et al., 2013) will be necessary the multi-scale characteristics and relations of the anchors, not only their characteristics and relation at one given scale. Similar to other pattern recognition problems in images, deep learning techniques offer faster recognition, as we recently proved for highway interchanges (Touya and Lokhat, 2020). But, as mentioned

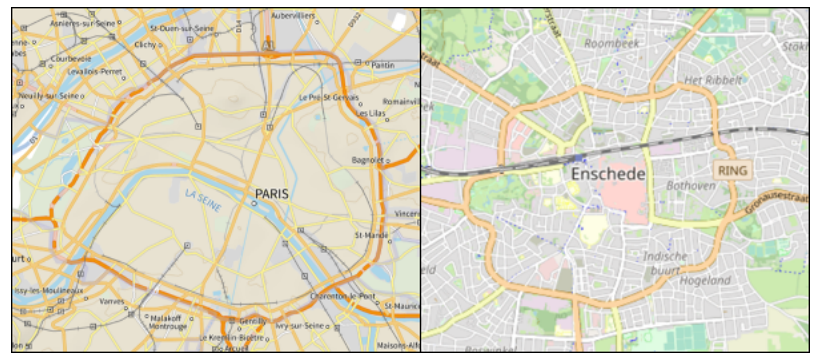

Figure 2. Two examples of very salient ring roads around Paris, France, and Enschede, the Netherlands. Both could be used as multi-scale anchors.

earlier, it is the multi-scale saliency that differentiates simple landmarks from anchors, so the recognition of anchors should use multiple scales as input. Attention-based mechanisms in neural networks enable the mutual and parallel processing of several images (Vaswani et al., 2017), and we plan to adopt such a framework to recognise the ideal anchors in a map.

\section{Progressive Multi-Scale Maps}

Although anchors are central in the LostInZoom project, we believe that focusing on anchors alone will not be sufficient to remove the desert fog. Map generalisation is also necessary to derive maps with progressive transformations, abstraction, including the abstraction of the anchors themselves. Progressive generalisation (Dumont et al., 2015) can be seen as a pragmatic and simpler version of continuous generalisation (van Oosterom et al., 2014), but both share the same goal of reducing the amount of transformations between maps at two consecutive scales (Dumont et al., 2020). Thus, the prototype multi-scale maps that will be designed to experiment with anchor-based zooming will benefit from state-of-the-art automated generalisation techniques to generate a large number of progressive maps, using the possibility in the recent versions of webmapping APIs to display maps even between the standard zoom levels. User studies will be carried out to assess how many generalised zoom levels are necessary for a map reader to 


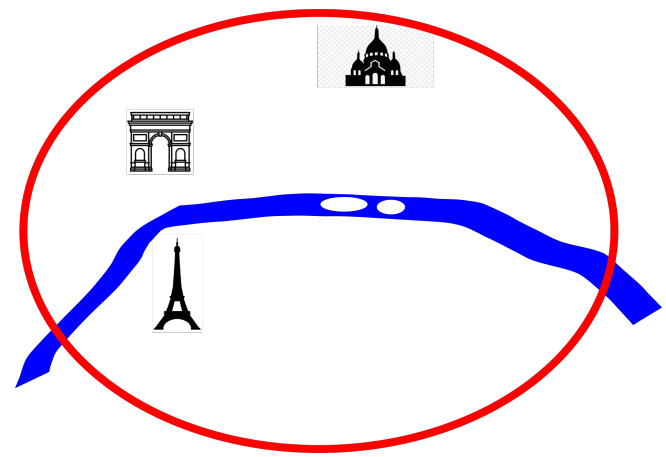

Figure 3. A naive cartography of Paris, France, based on anchors.

perceive smooth transformations between scales.

\section{Animations of Anchor-Based Zooming}

The final challenge is to compile all we have learned in previous steps to design a new zooming interaction technique (Bederson and Hollan, 1994) that makes use of anchors and the progressive generalisation of the map to help the user. Our proposition is to use a novel, landmark-aware animation during the zoom. Animations can help understanding the differences between two visualisations (Tversky et al., 2002), and have been successfully used with maps (Harrower and Fabrikant, 2008). Among the different types of animations, staged animations (Heer and Robertson, 2007), that define clear steps while zooming seem to be particularly adapted to our approach. To design the intermediate steps of the staged zooming animation, we plan to propose a kind of naive cartography - as largely defined by Egenhofer \& Mark (Egenhofer and Mark, 1995). Naive cartography is more concerned with the spatial and topological arrangement of anchors than with the precision of topographic maps. It deemphasizes precision in order to let the users focus on the anchors. Figure 3 shows an example of a naive cartography of the anchors that can be used during the staged animation. We also plan to adapt other types of multi-scale interactions to the anchor-based paradigm depending on the device and context of use: for instance, an anchor-based focus lens could be useful on small-display devices, while an anchor-based PolyZoom (Javed et al., 2012) could be useful on a wall-sized display.

\section{Conclusion}

All these challenges apply to the topographic maps used by the general public, but they are even more prominent with the maps used for crisis management, which need to be understood quickly, because of time constraints (Pierkot et al., 2019). We will team up with researchers on earthquake and tsunami risk management, to provide anchor-based multi-scale interactive maps dedicated to the management of these natural disasters, and to assess these propositions with real users.

The prototype softwares developed to find anchors in maps, to generalise progressive maps (Touya et al., 2019), or to create anchor-based zooming animations in a web map, will be released with an open source license. The material used for our spatial cognition surveys will also be released as open data to encourage future user surveys with interactive multi-scale maps.
Mixing map generalisation research with spatial cognition, map user issues, and human-computer interaction research, we hope that the LostInZoom project will overcome the current limitations of interactive multi-scale maps.

\section{Acknowledgements}

This project has received funding from the European Research Council (ERC) under the European Union's Horizon 2020 research and innovation programme (grant agreement No. 101003012).

\section{References}

Bederson, B. B. and Hollan, J. D., 1994. Pad++: A Zooming Graphical Interface for Exploring Alternate Interface Physics. In: Proceedings of the 7th Annual ACM Symposium on User Interface Software and Technology, UIST '94, ACM, New York, NY, USA, pp. 17-26. event-place: Marina del Rey, California, USA.

Bestgen, A.-K., Edler, D., Müller, K., Schulze, P., Dickmann, F. and Kuchinke, L., 2017. Where Is It (in the Map)? Recall and Recognition of Spatial Information. Cartographica 52(1), pp. 80-97.

Carral, D., Scheider, S., Janowicz, K., Vardeman, C., Krisnadhi, A. and Hitzler, P., 2013. An Ontology Design Pattern for Cartographic Map Scaling. In: P. Cimiano, O. Corcho, V. Presutti, L. Hollink and S. Rudolph (eds), The Semantic Web: Semantics and Big Data, Lecture Notes in Computer Science, Vol. 7882, Springer Berlin Heidelberg, pp. 76-93.

Couclelis, H., Golledge, R. G., Gale, N. and Tobler, W. 1987. Exploring the anchor-point hypothesis of spatial cognition. Journal of Environmental Psychology 7(2), pp. 99-122.

Dickmann, F., Edler, D., Bestgen, A.-K. and Kuchinke, L., 2017. Exploiting Illusory Grid Lines for Object-Location Memory Performance in Urban Topographic Maps. The Cartographic Journal 54(3), pp. 242-253.

Dumont, M., Touya, G. and Duchêne, C., 2015. Automated Generalisation of Intermediate Levels in a MultiScale Pyramid. In: Proceedings of 18th ICA Workshop on Generalisation and Multiple Representation, Rio de Janeiro, Brazil.

Dumont, M., Touya, G. and Duchêne, C., 2020. Designing multi-scale maps: lessons learned from existing practices. International Journal of Cartography 6(1), pp. 121-151.

Egenhofer, M. and Mark, D., 1995. Naive Geography. In: Spatial Information Theory A Theoretical Basis for GIS, Vol. 988, Springer, Semmering, Austria, pp. 1-15.

Harrower, M. and Fabrikant, S. I., 2008. The role of map animation in geographic visualization. In: M. Dodge (ed.), Geographic Visualization: Concepts, Tools and Applications, wiley edn, Chichester, UK, pp. 49-65.

Heer, J. and Robertson, G., 2007. Animated Transitions in Statistical Data Graphics. IEEE Transactions on Visualization and Computer Graphics 13(6), pp. 1240-1247.

Heinzle, F. and Anders, K.-H., 2007. Characterising Space via Pattern Recognition Techniques: Identifying Patterns in Road Networks. In: W. A. Mackaness, A. Ruas and T. Sarjakoski (eds), The Generalisation of Geographic Information : Models and Applications, Elsevier.

Javed, W., Ghani, S. and Elmqvist, N., 2012. Polyzoom: Multiscale and Multifocus Exploration in 2D Visual Spaces. In: Proceedings of the SIGCHI Conference on Human Factors in Computing Systems, CHI '12, ACM, New York, NY, USA, pp. 287-296. event-place: Austin, Texas, USA. 
Jul, S. and Furnas, G. W., 1998. Critical Zones in Desert Fog: Aids to Multiscale Navigation. In: Proceedings of the 11th Annual ACM Symposium on User Interface Software and Technology, UIST '98, ACM, New York, NY, USA, pp. 97-106. event-place: San Francisco, California, USA. Lüscher, P., Weibel, R. and Mackaness, W. A., 2008. Where is the Terraced House? On the Use of Ontologies for Recognition of Urban Concepts in Cartographic Databases. In: A. Ruas and C. Gold (eds), Headway in Spatial Data Handling, pp. 449-466.

Mackaness, W. A. and Edwards, G., 2002. The Importance of Modelling Pattern and Structure in Automated Map Generalisation. In: Proceedings of the Joint ISPRS/ICA Workshop on Multi-Scale Representations of Spatial Data, pp. 7-8.

Pierkot, C., Christophe, S. and Girres, J.-F., 2019. Exploring multiplexing tools for co-visualization in crisis units. In: 16th International Conference on Information Systems for Crisis Response and Management (ISCRAM 2019), Valencia, Spain.

Rosenholtz, R., Li, Y. and Nakano, L., 2007. Measuring visual clutter. Journal of Vision.

Roth, R. E., 2013. Interactive maps: What we know and what we need to know. Journal of Spatial Information Science 6, pp. 59-115.

Roth, R. E., Çöltekin, A., Delazari, L., Filho, H. F., Griffin, A., Hall, A., Korpi, J., Lokka, I., Mendonça, A., Ooms, K. and van Elzakker, C. P. J. M., 2017. User studies in cartography: opportunities for empirical research on interactive maps and visualizations. International Journal of Cartography 3(sup1), pp. 61-89.

Sorrows, M. E. and Hirtle, S. C., 1999. The Nature of Landmarks for Real and Electronic Spaces. In: Proceedings of the International Conference on Spatial Information Theory: Cognitive and Computational Foundations of Geographic Information Science, Lecture Notes in Computer Science, Springer-Verlag, London, UK, UK, pp. 3750.

Touya, G., 2019. Finding the Oasis in the Desert Fog? Understanding Multi-Scale Map Reading. In: Proceedings of the ICA Workshop on Abstraction, Scale and Perception, Tokyo, Japan.

Touya, G. and Lokhat, I., 2020. Deep Learning for Enrichment of Vector Spatial Databases: Application to Highway Interchange. ACM Transactions on Spatial Algorithms and Systems 6(3), pp. 21. Publisher: ACM.

Touya, G., Bucher, B., Falquet, G., Jaara, K. and Steiniger, S., 2014. Modelling Geographic Relationships in Automated Environments. In: D. Burghardt, C. Duchêne and W. Mackaness (eds), Abstracting Geographic Information in a Data Rich World, Springer, Berlin Heidelberg, pp. 5382.

Touya, G., Lokhat, I. and Duchêne, C., 2019. CartAGen: an Open Source Research Platform for Map Generalization. Proceedings of the ICA 2, pp. 1-9.

Tversky, B., Morrison, J. B. and Betrancourt, M., 2002. Animation: can it facilitate? International Journal of Human-Computer Studies 57(4), pp. 247-262.

van Oosterom, P., Meijers, M., Stoter, J. and Šuba, R., 2014. Data Structures for Continuous Generalisation: tGAP and SSC. In: D. Burghardt, C. Duchêne and W. Mackaness (eds), Abstracting Geographic Information in a Data Rich World, Lecture Notes in Geoinformation and Cartography, Springer International Publishing, pp. 83-117.

Vaswani, A., Shazeer, N., Parmar, N., Uszkoreit, J., Jones, L., Gomez, A. N., Kaiser, L. and Polosukhin, I., 2017. Attention Is All You Need. arXiv:1706.03762 [cs]. 